Research Article

\title{
Performance Evaluation of Malaria Pf/Pv Combo Test Kit at Highly Malaria-Endemic Area, Southern Ethiopia: A Cross-Sectional Study
}

\author{
Temesgen Eticha ${ }^{(D},{ }^{1}$ Tewodros Tamire, ${ }^{2}$ and Temesgen Bati ${ }^{3}$ \\ ${ }^{1}$ Department of Medical Laboratory Sciences, College of Health Sciences and Medicine, Wolaita Sodo University, Sodo, Ethiopia \\ ${ }^{2}$ Microbiology unit, Tikur Anbessa Specialized Hospital, Addis Ababa, Ethiopia \\ ${ }^{3}$ School of public health, College of Health Sciences and Medicine, Wolaita Sodo University, Sodo, Ethiopia
}

Correspondence should be addressed to Temesgen Eticha; etichatemesgen@gmail.com

Received 21 May 2020; Revised 5 August 2020; Accepted 26 August 2020; Published 7 September 2020

Academic Editor: Shyam Sundar

Copyright (c) 2020 Temesgen Eticha et al. This is an open access article distributed under the Creative Commons Attribution License, which permits unrestricted use, distribution, and reproduction in any medium, provided the original work is properly cited.

\begin{abstract}
Background. Malaria rapid diagnostic tests (RDTs) are alternative diagnostic methods that have enabled reliable biological diagnostic testing in all situations where previously only clinical diagnosis was available. Varying diagnostic accuracy of malaria RDTs makes policymakers confused while choosing malaria test kits for their country. Objective. The aim of this study was to evaluate the diagnostic performance of currently being used malaria RDT in Southern Ethiopia. Methods. A cross-sectional study design was conducted from October 1 to December 15, 2016. A total of 160 patients were included in the study. Finger-prick blood sample was obtained from study subjects for the RDT test and microscopic examination. Collected data were entered and analyzed using SPSS version 20.0. Result. The test kit evaluated had an overall sensitivity, specificity, PPV, and NPV of 97.44\%, 93.67\%, $93.83 \%$, and $97.37 \%$, respectively, to detect the presence or absence of malaria. Sensitivity and specificity of the kit for $P$. falciparum detection were $63.27 \%$ and $94.3 \%$ and for $P$. vivax detection were $86.96 \%$ and $95.62 \%$, respectively. The agreement between microscopy and RDT for specific identification of malaria species was moderate with a kappa value of 0.568 . Conclusion. The overall performance of the kit was below the WHO standard. Further study on a large sample size is recommended to be carried out in the study area to use the test kit instead of microscopy for malaria diagnosis. Providing training on quality malaria laboratory diagnosis and availing necessary supplies for malaria diagnosis shall also be considered.
\end{abstract}

\section{Introduction}

In Ethiopia, malaria is the leading cause of morbidity and mortality. Almost $75 \%$ of the country is malarious, and an estimated 51 million people (68\% of the population) live in areas at risk of malaria [1]. It is a parasitic disease that is transmitted to humans by mosquitoes of the genus Anopheles [2]. It is one of the few diseases which have a quick, simple, and accurate biological diagnostic method even in a low-technology setting [3].

The nonspecific nature of signs and symptoms of malaria made a clinical diagnosis of the disease unreliable [4]. Microscopy remains the standard and preferred method of diagnosis compared to other methods despite its apparent drawbacks especially in sub-Saharan African hospitals as it needs significant technical skills and good-quality reagents $[4,5]$.

RDTs are alternate diagnostic methods which enabled reliable biological diagnostic testing in situations where previously only clinical diagnoses were available, although it has varying diagnostic accuracy across different geographical regions $[6,7]$. Their adoption needs to be guided by local sensitivity tests, to detect functional problems due to exposure to heat, humidity, and manufacturing faults $[8,9]$.

The ability of Care Start ${ }^{\mathrm{TM}}$ Malaria Pf/Pv Combo test to diagnose Plasmodium malaria was very good, with $99.8 \%$ sensitivity and $97.7 \%$ specificity, according to the study conducted on acute febrile patients visiting the Felegeselam 
Health Center, North Ethiopia [10]. Similarly, sensitivity and specificity of the Care Start ${ }^{\mathrm{TM}}$ Malaria Pf/Pv Combo test were $98.5 \%$ and $98.0 \%$ for the diagnosis of $P$. falciparum and $P$. vivax infections according to a study performed in Afar region, Northeast Ethiopia [11].

Even though Arba Minch and adjacent hot rural areas are one of the most highly malarious places in Ethiopia and using malaria RDTs as an alternative diagnostic tool, its performance was not evaluated so far. This study aimed at evaluating the diagnostic performance of malaria RDT by determining its sensitivity and specificity compared with microscopy to help local malaria eradication program presenting the actual performance of currently used malaria RDT.

\section{Materials and Methods}

2.1. Study Area. The study was conducted in Arba Minch General Hospital (AGH) and Arba Minch Health Center. Arba Minch town is located in Gamo Gofa Zone of the Southern Nations, Nationalities, and People's Region (SNNPR) about 500 kilometers south of Addis Ababa. It is found at an altitude of 1200-1300 meters above sea level with an average annual temperature of $29.7^{\circ} \mathrm{C}$ and rainfall of $700 \mathrm{~mm}$ [12].

2.2. Study Design and Period. A cross-sectional study design was employed from October 1 to December 15, 2016.

2.3. Study Participants. The study participants were all individuals who came to AGH and Arba Minch Health Center being suspected of malaria and have a request paper for malaria diagnosis at the respective laboratory unit.

2.4. Inclusion Criteria. All age group patients with clinical signs and symptoms of malaria who presented to AGH and health center laboratory department for confirmation during the study periods were eligible for enrollment into the study.

2.5. Exclusion Criteria. Clients who do not fulfill the inclusion criteria and refused participation were excluded from the study.

2.6. Sample Size and Sampling Technique. Assuming a confidence level of $95 \%$, an error risk of 1.96, an expected minimum sensitivity and specificity of all RDTs of $95 \%$, compared with microscopy according to the World Health Organization [13], and a margin of error of $5 \%$ plus an additional $10 \%$ of the sample to account for invalid and unclear results, a minimum sample of 80 participants were recruited. According to this assumption, a total of 160 study subjects ( 80 malaria-positive and 80 malaria-negative) were included in this study.

A convenient sampling technique was employed to obtain those study subjects. $n=\left(\left(z^{2}\right) p(1-p) / d^{2}\right)=80$ (including $10 \%$ to account for invalid and unclear results), where $n$ is the sample size, $z$ is the $95 \%$ confidence interval (1.96), $d$ is the margin of error (5\%), and $p$ is the sensitivity or specificity (95\%).

2.7. Data Gathering Procedures. Questionnaires were developed for the purpose of simple sociodemographic data. Formats were used for reporting parasite density and the laboratory result of malaria status by microscopy and RDT. They were prepared originally in English after reviewing relevant literature and then translated to Amharic. Pretest of the questionnaire for the clarity and consistency of questions was performed, and the necessary correction was made based on the findings of the pretest.

2.8. Sample Collection and Processing. A blood sample was obtained from eligible study subjects visiting Arba Minch General Hospital and Arba Minch Health Center in the specified study period, having signs and symptoms of malaria, and sent to laboratory diagnosis. From each study participant, a finger-prick blood sample was collected for malaria testing with both Care Start ${ }^{\mathrm{TM}}$ RDT (Cat No.G0161 T) and microscope. Two blood films were prepared; the first blood film was processed at a hospital or health center laboratory setup as a routine way, and the result at such facility setup was used as primary reading. Such primary result was recorded separately for the research purpose according to the prelabeled identification number. The second blood film was dried and fixed (the thin film) and examined at Arba Minch College of Health Science teaching laboratory by the principal investigator for quality assurance purposes. Any discrepancy between primary reading and secondary reading was cleared by third-person final reading. The Care Start ${ }^{\mathrm{TM}}$ RDT test was performed at the site of sample collection by different laboratory personnel so that the one who did the microscopy do not know the result of the RDT.

\subsection{Laboratory Procedures}

2.9.1. Rapid Diagnostic Testing for Malaria. The available RDT kit was used in this study according to the manufacturer's instructions. It is used for the qualitative detection of antigens produced by $P$. falciparum and $P$. vivax. These antigens are the histidine-rich protein -2 (PfHRP-2) and lactate dehydrogenase (LDH).

2.9.2. Microscopy. Blood smears were stained with $10 \%$ Giemsa and examined using 100X oil immersion objective lens of a light microscope by two independent laboratory technologists (one at the hospital/health center and the other at the teaching school laboratory (AMCHS)) who are blinded to each other's results.

2.9.3. Parasite Density. Parasite density estimation was performed by measuring the level of infection in red blood cells by examination of a thin blood film which is confirmed 
to be more accurate [14]. To quantify malaria parasites against RBCs, parasitized RBCs among 500-2,000 RBCs on the thin smear were counted, and the result is expressed as a percentage of parasitemia.

2.9.4. Methods of Data Analysis. Data collected from this study were entered and analyzed using SPSS version 20 . The data include participant demographic information, clinical signs and symptoms, parasite type, previous treatment history, and RDT result. Sensitivity, specificity, positive predictive values (PPVs), and negative predictive values (NPVs) of the Care Start ${ }^{\mathrm{TM}}$ Malaria RDT were calculated. Kappa value was calculated to determine the agreement between the results of microscopy and the diagnostic test kit.

2.9.5. Data Quality Management. Fresh blood samples were transferred directly to the sample pad by the provided sample applicator. All Care Start ${ }^{\mathrm{TM}}$ malaria test kits were labeled with the patient ID number, and the procedure and the result recording period were according to the manufacturer's instruction. To eliminate observer bias, quality control was performed by the principal investigator at Arba Minch College of Health Science teaching laboratory blindly repeating all test results at the facility level.

\section{Results}

In this study, a total of 160 study participants suspected of malaria were examined for malaria parasites by thick/thin blood smear microscopy and Care Start ${ }^{\mathrm{TM}}$ combo RDT test kit. Of the total participants, $89(55.6 \%)$ were males and 71 (44.4\%) were females with ages ranging from 1 year to 70 years with a mean age of 24 and a median of 21 (Table 1).

In this study, microscopy and Care Start ${ }^{\mathrm{TM}}$ malaria RDT gave similar $P$. falciparum test result for 31 study participants, $P$. vivax test result for 20 study participants, and mixed infection with $P$. falciparum and $P$. vivax for only 5 study participants. As presented in Table 2, surprisingly RDT gave mixed infection for 13 study participants which were only P. falciparum by microscopy.

Of the entire 160 blood smear slides eligible for analysis, microscopy detected malaria parasites in $80(50 \%)$ blood smear samples. Among microscopically examined blood smear samples of study participants, 51 (63.75\%) were infected with $P$. falciparum, 23 (28.75\%) were infected with $P$. vivax, and the remaining six $(7.50 \%)$ had mixed infection with $P$. falciparum and $P$. vivax (Figure 1). No study participants were identified as positive with $P$. ovale and P. malariae by malaria microscopy.

Taking a thick blood smear as a gold standard test for malaria, the overall sensitivity and specificity of Care Start ${ }^{\mathrm{TM}}$ RDT were found to be $97.44 \%$ (95\% CI $=91.04 \%-99.69 \%)$ and $93.67 \%$ (95\% CI $=85.84 \%-97.91 \%)$, respectively. The PPV and the NPV of the device were found to be $93.83 \%$ $(95 \% \mathrm{CI}=86.18 \%-97.97 \%)$ and $97.37 \%$ (95\% CI $=90.82 \%-$ $99.68 \%$ ), respectively. The agreement between the light microscopy and Care Start ${ }^{\mathrm{TM}}$ RDT to detect the presence or absence of the malaria parasite has a kappa value of 0.877 (Table 3).

The Care Start $^{\mathrm{TM}}$ RDT test kit was $63.27 \% \quad(95 \%$ $\mathrm{CI}=48.29 \%-76.58 \%) \quad$ sensitive and $94.50 \% \quad(95 \%$ $\mathrm{CI}=88.40 \%-97.95 \%)$ specific to detect $P$. falciparum malaria. The PPV and NPV of Care Start ${ }^{\mathrm{TM}}$ RDT test kit to diagnose $P$. falciparum were $83.78 \%$ and $85.12 \%$, respectively. The corresponding sensitivity and specificity of Care Start $^{\mathrm{TM}}$ for the diagnosis P. vivax malaria were $86.96 \%$ (95\% $\mathrm{CI}=66.41 \%-97.22 \%) \quad$ and $\quad 95.62 \% \quad(95 \% \quad \mathrm{CI}=90.71 \%-$ 98.38\%), respectively, with $76.92 \%$ PPV and $97.76 \%$ NPV. The sensitivity, specificity, PPV, and NPV of Care Start ${ }^{\mathrm{TM}}$ for the diagnosis of mixed infection (P. falciparum and P. vivax) were $83.33 \%, 91.56 \%, 27.78 \%$, and $99.30 \%$, respectively. Accordingly, the overall agreement between light microscopy and Care Start ${ }^{\mathrm{TM}}$ malaria RDT test kit for specific identification of malaria species has a kappa value of 0.568 (Table 4).

On analysis of the diagnostic performance of the kit used by percent parasitemia, generally, the sensitivity of the kit increased when the percent parasitemia increased in the individual study subject. Assessment of sensitivities at percent parasitemia threshold below $0.049 \%$ was not displayed because there is no positive test result by microscopy within that percent parasitemia range. Starting from 1.01\% parasitemia up to the maximum percent parasitemia (2.85\%), the Care Start ${ }^{\mathrm{TM}}$ RDT showed $100 \%$ sensitivity. Association of percent parasite load with RDT and microscopy result is presented in Table 5 .

Care Start ${ }^{\mathrm{TM}}$ Combo test for the detection of P. falciparum had a sensitivity of $100 \%$ for study participants with percent parasitemia $>1.51 \%$. As indicated in Table 6 , the sensitivity of the test kit declined from $80.77 \%$ (for P. falciparum) and $90.91 \%$ (for P. vivax) to $46.67 \%$ and $75.00 \%$, respectively, even though the percent parasitemia increased (Table 6).

\section{Discussion}

Using RDT devices to test the presence or absence of the malaria parasite has many advantages, especially in countries such as Ethiopia where the majority of the population live in rural parts of the country where access to electricity and infrastructures are limited; using RDTs as a diagnostic testing method and treatment monitoring method has a great advantage. Since the performance of these diagnostic testing methods may vary from population to population even in the same country, their diagnostic performance shall be tested locally to check their agreement with the WHO minimal sensitivity and specificity for any RDTs.

In this study, Care Start ${ }^{\mathrm{TM}}$ Combo test showed an overall sensitivity, specificity, PPV, and NPV of 97.44\%, 93.67\%, $93.83 \%$, and $97.37 \%$, respectively. It showed a better performance when compared with the study conducted in Butajira area, south-central Ethiopia, with overall sensitivity and specificity for the diagnosis of malaria of $90.8 \%$ and $82.7 \%$, respectively [15]. The variation in the result may be due to the difference in the method used (facility and community survey), with a greater sample size, as they 
Table 1: Age group distribution of the study participants, Arba Minch, 2016.

\begin{tabular}{lccc}
\hline Age group & Frequency & Percentage & Microscopy positive result \\
\hline$<5$ years & 16 & 10 & 9 \\
$6-15$ years & 31 & 19.4 & 24 \\
$16-30$ years & 72 & 45 & 37 \\
$31-45$ years & 25 & 15.6 & 9 \\
$>45$ years & 16 & 10 & 1 \\
Total & 160 & 100 & 80 \\
\hline
\end{tabular}

TABle 2: Comparison of Care Start ${ }^{\mathrm{TM}}$ malaria RDT with microscopy for the identification of malaria species, Arba Minch, 2015.

\begin{tabular}{|c|c|c|c|c|c|}
\hline & \multicolumn{5}{|c|}{ Malaria species seen by microscopy } \\
\hline \multirow{5}{*}{ Malaria species detected by RDT } & & P. falciparum & P. vivax & Mixed & Total \\
\hline & P. falciparum & 31 & 3 & 0 & 34 \\
\hline & P. vivax & 3 & 20 & 1 & 24 \\
\hline & Mixed & 13 & 0 & 5 & 18 \\
\hline & Total & 47 & 23 & 6 & 76 \\
\hline
\end{tabular}

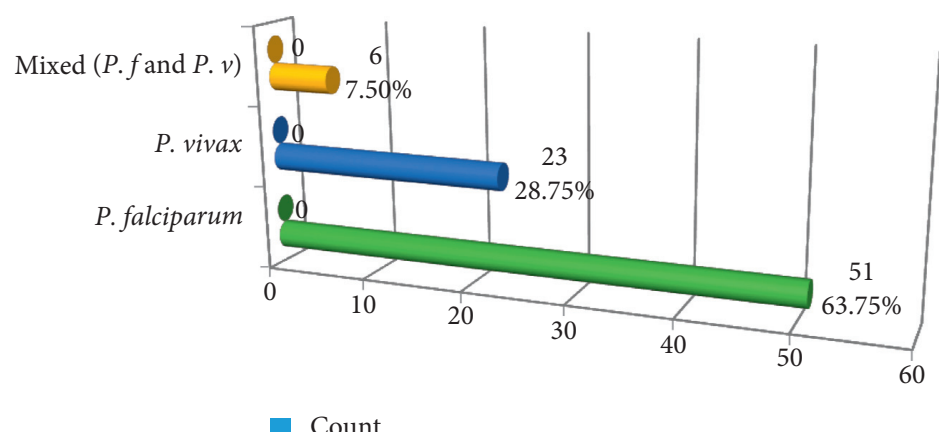

FIgURE 1: Malaria species prevalence among microscopically confirmed positive cases, Arba Minch, 2016.

TABLE 3: Overall performance characteristics of Care Start ${ }^{\mathrm{TM}}$ in comparison with the standard light microscopy, Arba Minch, 2016.

\begin{tabular}{lr}
\hline Performance characteristics & Care Start $^{\mathrm{TM}}$ malaria $^{-}$ \\
\hline Sensitivity (95\% CI) & $97.44 \%(91.04 \%-99.69 \%)$ \\
Specificity (95\% CI) & $93.67 \%(85.84 \%-97.91 \%)$ \\
Positive predictive value (95\% CI) & $93.83 \%(86.18 \%-97.97 \%)$ \\
Negative predictive value (95\% CI) & $97.37 \%(90.82 \%-99.68 \%)$ \\
\hline
\end{tabular}

TABle 4: Performance characteristics of Care Start ${ }^{\mathrm{TM}}$ by species identified in comparison with the standard light microscopy, Arba Minch, 2016.

\begin{tabular}{lcccc}
\hline Parasite type & Sensitivity $(95 \%$ CI $)$ & Specificity $(95 \%$ CI $)$ & PPV (95\% CI) & NPV (95\% CI) \\
\hline P. falciparum & $63.27 \%(48.29-76.58)$ & $94.50 \%(88.40-97.95)$ & $83.78 \%(67.99-93.81)$ & $85.12 \%(77.51-90.94)$ \\
P. vivax & $86.96 \%(66.41-97.22)$ & $95.62 \%(90.71-98.38)$ & $76.92 \%(56.35-91.03)$ & $97.76 \%(93.60-99.54)$ \\
Mixed infection & $83.33 \%[35.88-99.58 \%]$ & $91.56 \%(86.00-95.43)$ & $27.78 \%(9.69-53.48)$ & $99.30 \%(96.14-99.98)$ \\
Overall performance & $97.44 \%(91.04-99.69)$ & $93.67 \%(85.84-97.91)$ & $93.83 \%(86.18-97.97)$ & $97.37 \%(90.82-99.68)$ \\
\hline
\end{tabular}

included every febrile case in the study. In contrast, when compared with the study conducted on acute febrile patients visiting Felegeselam Health Center with $99.8 \%$ sensitivity and $97.7 \%$ specificity [10], our device showed poor overall performance.

Our device has shown almost comparable overall performance with studies carried out in Northwest Ethiopia with an overall sensitivity of $95 \%$ and specificity of $94.2 \%$ [4], at Serbo Health Center in Jimma Zone, Southwestern Ethiopia, with an overall sensitivity of $95.8 \%$ and specificity of $100 \%$ [1], and in Northwestern Tigray with a sensitivity of 95.4\% [16]. This comparable performance may be due to almost a similar sampling technique, testing procedures, and analysis.

This study has also tried to evaluate the performance of the Care Start ${ }^{\mathrm{TM}}$ RDT in detecting different species of malaria 
TABle 5: Association between the sensitivity of Care Start ${ }^{\mathrm{TM}}$ and percent parasitemia, Arba Minch, 2016.

\begin{tabular}{lr}
\hline Range of percent parasitemia & Sensitivity (95\% CI) \\
\hline$<0.049 \%$ & ND \\
$0.05 \%-0.50 \%$ & $97.44 \%(86.52-99.94)$ \\
$0.51 \%-1.00 \%$ & $96.15 \%(80.36-99.90)$ \\
$1.01 \%-1.50 \%$ & $100 \%(59.04-100)$ \\
$1.51 \%-2.00 \%$ & $100 \%(15.81-100)$ \\
$>2.01 \%$ & $100 \%(39.76-100)$ \\
\hline
\end{tabular}

Table 6: Sensitivity and specificity of Care Start ${ }^{\mathrm{TM}}$ Malaria Pf/Pv Combo test for the diagnosis of malaria species infections at different levels of percent parasitemia, Arba Minch, 2016.

\begin{tabular}{|c|c|c|c|c|}
\hline \multirow{2}{*}{$\begin{array}{l}\text { Parasite type } \\
\text { Range of percent parasitemia }\end{array}$} & \multicolumn{2}{|c|}{ Sensitivity } & \multicolumn{2}{|c|}{ Specificity } \\
\hline & P. falciparum & P. vivax & P. falciparum & P. vivax \\
\hline$<0.049 \%$ & ND & ND & ND & ND \\
\hline $0.05 \%-0.50 \%$ & $80.77 \%$ & $90.91 \%$ & $92.31 \%$ & $88.89 \%$ \\
\hline $0.51 \%-1.00 \%$ & $46.67 \%$ & $75.00 \%$ & $81.82 \%$ & $94.74 \%$ \\
\hline $1.01 \%-1.50 \%$ & $0 \%$ & $100 \%$ & $100 \%$ & $100 \%$ \\
\hline $1.51 \%-2.00 \%$ & $100 \%$ & ND & ND & ND \\
\hline$>2.01 \%$ & $100 \%$ & $100 \%$ & $100 \%$ & $100 \%$ \\
\hline
\end{tabular}

parasite. In this study, the sensitivity and specificity of Care Start $^{\mathrm{TM}}$ RDT for the diagnosis of P. falciparum are $63.27 \%$ and $94.3 \%$, respectively, which showed very poor performance when compared to other studies with sensitivity and specificity of $99.4 \%$ and $98 \%$ in Wondo Genet [17], 98.5\% and $98.0 \%$ in Afar region [11], and $85.6 \%$ and $92 \%$ in Oromia Regional State [18], respectively. Variations in test sensitivity between these studies may be due to variations in epidemiologic characteristics of the study population, level of parasitemia, test methodology, and skill of microscopists [19].

Even though the sensitivity and specificity of Care Start ${ }^{\mathrm{TM}}$ RDT for the diagnosis of $P$. vivax were better than those for P. falciparum, with $86.96 \%$ and $95.62 \%$, respectively, it has almost comparable performance with the study carried out at three health centers in Jimma Zone, Oromia Regional State [18]. It showed poor performance when compared with other studies in Wondo Genet (99.4\% and 98.2\%) [17] and Afar (100\% and 99.6\%) [11]. Although the manufacturer's instructions were strictly followed, the poor performance of this RDT kit in the current study could be due to the high false-positive results possibly because of the persistent nature of HRP-2 [20] and variation of the geographical regions [7].

Accordingly, even though there was a very good agreement between the light microscopy and Care Start ${ }^{\mathrm{TM}}$ RDT to detect the presence or absence of malaria parasite with a kappa value of 0.877 , the overall agreement between light microscopy and Care Start ${ }^{\mathrm{TM}}$ malaria RDT for specific identification of malaria species is very poor with a Kappa value of 0.568 .

The performance of Care Start ${ }^{\mathrm{TM}}$ RDT was noted to be significantly influenced by the level of percent parasitemia. In this study, the percent parasitemia ranged from $0.05 \%$ to $2.85 \%$. The sensitivity and specificity of the RDT increased when the percent parasitemia increased in the individual study subjects. A similar scenario was observed in other studies $[9,11,21,22]$. In addition, the chance of malaria RDT kit used to show mixed infection for study participants who had only P. falciparum was very high. 13 (8.12\%) study participants who had $P$. falciparum only by the standard microscopy were found to have mixed infection by the kit used. A similar finding was observed in a study performed in Madagascar [23] where the kit used (SD Bioline Malaria Ag $\mathrm{Pf} / \mathrm{Pan}$ ) showed mixed infection for those detected as high parasite density $P$. falciparum. This may result in unnecessary drug and resource expenditure in areas where only RDT kit is being utilized as a means of malaria diagnosis.

The main limitation of the study was not using PCR as a reference method, since microscopy has lower sensitivity even when compared with loop-mediated isothermal amplification (LAMP) [24], and obviously, its sensitivity decreases with decreased parasite density.

\section{Conclusion}

Malaria RDT kits may be used in areas where there are no trained personnel, electricity, necessary equipment, and reagents which are necessary for malaria microscopy. Since the performance of RDT may vary with various factors such as loss of integrity of kits during transportation, different climate conditions, and/or inappropriate storage condition, frequent local sensitivity testing shall be carried out.

In this study, the performance of the currently used malaria RDT kit was poor for accurate diagnosis of malaria. It has also a drawback in correctly identifying malaria species of $P$. falciparum and P. vivax. So, further studies should be carried out to use the Care Start ${ }^{\mathrm{TM}}$ Malaria Pf/Pv Combo test kit instead of microscopy for the diagnosis of malaria. Providing training on quality malaria laboratory diagnosis for the staff and availing necessary supplies for malaria diagnosis shall also be considered. Gamo Gofa Zone Health 
Department should select and purchase the right type of malaria RDT which displays good performance by conducting area-specific performance evaluation tests.

\section{Data Availability}

Data used to support the finding of this study are available from the corresponding author upon request.

\section{Ethical Approval}

Ethical permission to perform the study was obtained from the Department of Research and Ethical Review Committee (DRERC) of Medical Laboratory Science, School of Allied Health Science, College of Health Science, Addis Ababa University.

\section{Consent}

Informed written consent was obtained from all study participants or caregivers/parents in the case of children.

\section{Conflicts of Interest}

The authors declare that there are no conflicts of interest regarding the publication of this paper.

\section{Acknowledgments}

The authors would like to thank Addis Ababa University for the financial support provided to accomplish this work.

\section{References}

[1] Z. Mekonnen, S. Ali, G. Belay, S. Suleman, and S. Chatterjee, "Evaluation of the performance of care start malaria $\mathrm{Pf} / \mathrm{Pv}$ combo rapid diagnostic test for the diagnosis of malaria in Jimma, Southwestern Ethiopia," Acta Tropica, vol. 113, no. 3, pp. 285-288, 2009.

[2] J. C. Mouatcho and J. P. D. Goldring, "Malaria rapid diagnostic tests: challenges and prospects," Journal of Medical Microbiology, vol. 62, no. 10, pp. 1491-1505, 2013.

[3] E. A. Ashley, M. Touabi, M. Ahrer et al., "Evaluation of three parasite lactate dehydrogenase-based rapid diagnostic tests for the diagnosis of falciparum and vivax malaria," Malaria Journal, vol. 8, no. 1, p. 241, 2009.

[4] B. Moges, B. Amare, Y. Belyhun et al., "Comparison of care start HRP2/pLDH COMBO rapid malaria test with light microscopy in North-west Ethiopia," Malaria Journal, vol. 11, p. 234, 2012.

[5] A. H. Kashif, G. K. Adam, A. A. Mohmmed, S. E. Elzaki, A. M. AbdelHalim, and I. Adam, "Reliability of rapid diagnostic test for diagnosing peripheral and placental malaria in an area of unstable malaria transmission in Eastern Sudan," Diagnostic Pathology, vol. 8, p. 59, 2013.

[6] S. Kim, S. Nhem, D. Dourng, and D. Ménard, "Malaria rapid diagnostic test as a point-of-care test: study protocol for evaluating the VIKIA ${ }^{\circledR}$ malaria Ag Pf/Pan," Malaria Journal, vol. 14, no. 1, p. 114, 2015.

[7] T. A. Abeku, M. Kristan, C. Jones et al., "Determinants of the accuracy of rapid diagnostic tests in malaria case management: evidence from low and moderate transmission settings in the East African highlands," Malaria Journal, vol. 7, no. 1, p. 202, 2008.

[8] World Health Organization, "Evaluation of rapid diagnostic tests: malaria," Nature Reviews Microbiology, vol. 4, pp. S34-S38, 2006.

[9] U. M. Sani, N. M. Jiya, and H. Ahmed, "Evaluation of malaria rapid diagnostic test among febrile children in Sokoto, Nigeria," International Journal of Medicine and Medical Sciences, vol. 3, no. 5, pp. 434-440, 2013.

[10] T. Hailu and T. Kebede, "Assessing the performance of care start malaria Pf/Pv Combo Test against thick blood film in the diagnosis of malaria in northwest Ethiopia," The American Journal of Tropical Medicine and Hygiene, vol. 90, no. 6, pp. 1109-1112, 2014.

[11] M. Chanie, B. Erko, A. Animut, and M. Legesse, "Performance of Care Start ${ }^{\mathrm{TM}}$ Malaria Pf/Pv Combo test for the diagnosis of Plasmodium falciparum and Plasmodium vivax infections in the Afar region, North East Ethiopia," Ethiopian Journal of Health Development, vol. 25, no. 3, pp. 207-211, 2011.

[12] A. Astatkie, "Knowledge and practice of malaria prevention methods among residents of Arba Minch town and Arba Minch Zuria district, Southern Ethiopia," Ethiopian Journal of Health Sciences, vol. 20, no. 3, pp. 185-193, 2010.

[13] WHO, Malaria Diagnosis New Perspectives Report of a Joint WHO/USAID Informal Consultation, WHO, Geneva, Switzerland, 2000.

[14] B. M. Greenwood and J. R. M. Armstrong, "Comparison of two simple methods for determining malaria parasite density," Transactions of the Royal Society of Tropical Medicine and Hygiene, vol. 85, no. 2, pp. 186-188, 1991.

[15] W. D. Adugna Woyessa, A. Ahmed, and B. Lindtjørn, "Evaluation of Care Start ${ }^{\mathrm{TM}}$ malaria $\mathrm{Pf} / \mathrm{Pv}$ Combo test for Plasmodium falciparum and Plasmodium vivax malaria diagnosis in Butajira area, South-Central Ethiopia," Malaria Journal, vol. 12, no. 1, p. 218, 2013.

[16] D. G. Feleke, S. Tarko, and H. Hadush, "Performance comparison of CareStart ${ }^{\mathrm{TM}} \mathrm{HRP} 2 / \mathrm{pLDH}$ combo rapid malaria test with light microscopy in North-Western Tigray, Ethiopia: a cross-sectional study," BMC Infectious Diseases, vol. 17, no. 1, p. 399, 2017.

[17] B. Sharew, M. Legesse, A. Animut, D. Jima, G. Medhin, and B. Erko, "Evaluation of the performance of care start malaria $\mathrm{Pf} / \mathrm{Pv}$ combo and paracheck Pf tests for the diagnosis of malaria in Wondo Genet, Southern Ethiopia," Acta Tropica, vol. 111, no. 3, pp. 321-324, 2009.

[18] R. A. Ashton, T. Kefyalew, G. Tesfaye et al., "Performance of three multi-species rapid diagnostic tests for diagnosis of Plasmodium falciparum and Plasmodium vivax malaria in Oromia Regional State, Ethiopia," Malaria Journal, vol. 9, p. 297, 2010.

[19] C. Wongsrichanalai, W. H. Wernsdorfer, S. Muth, A. Sutamihardja, and M. J. Barcus, "A review of malaria diagnostic tools: microscopy and rapid diagnostic test (RDT)," The American Journal of Tropical Medicine and Hygiene, vol. 77, no. 6_Suppl, pp. 119-127, 2007.

[20] D. J. Kyabayinze, J. K. Tibenderana, G. W. Odong, J. B. Rwakimari, and H. Counihan, "Operational accuracy and comparative persistent antigenicity of HRP2 rapid diagnostic tests for Plasmodium falciparum malaria in a hyperendemic region of Uganda," Malaria Journal, vol. 7, no. 1, p. 221, 2008.

[21] J. Maltha, P. Gillet, L. Cnops et al., "Evaluation of the rapid diagnostic test SDFK40 (Pf-pLDH/pan-pLDH) for the diagnosis of malaria in a non-endemic setting," Malaria Journal, vol. 10, no. 1, p. 7, 2011. 
[22] I. Van Den Broek, J.-P. Guthmann, B. Angarita et al., "Evaluation of three rapid tests for diagnosis of $P$. falciparum and $P$. vivax malaria in Colombia," The American Journal of Tropical Medicine and Hygiene, vol. 75, no. 6, pp. 1209-1215, 2006.

[23] R. K. Mehlotra, R. E. Howes, E. Y. Cramer et al., "Plasmodium falciparum parasitemia and band Sensitivity of the SD bioline malaria Ag P.f/Pan rapid diagnostic test in Madagascar," The American Journal of Tropical Medicine and Hygiene, vol. 100, no. 5, pp. 1196-1201, 2019.

[24] S. Girma, J. Cheaveau, A. Naser Mohon et al., "Prevalence and epidemiological characteristics of asymptomatic malaria based on ultrasensitive diagnostics: a cross sectional study," Clinical Infectious Disease, vol. 69, no. 6, pp. 1003-1010, 2019. 